\title{
Six month evaluation of thiopropazate hydrochloride in tardive dyskinesia
}

\author{
J. S Y D N E Y S M I T H A D L. G. K I L OH \\ From the Neuropsychiatric Institute, Prince Henry Hospital, Little Bay, and \\ Department of Psychiatry, University of New South Wales, Sydney, New South Wales, Australia
}

S UMMARY Using a blind evaluation of cinematographic films of patients suffering from tardive dyskinesia we found that thiopropazate hydrochloride in a dosage up to $30 \mathrm{mg}$ daily was effective in reducing the severity of the dyskinesia in most patients. The overall improvement in the group of patients studied was not significant after one or three months of therapy but was significant after six months of treatment. The administration of thiopropazate hydrochloride over a six month period did not appear to aggravate the underlying pathophysiology so that the drug could be considered likely to be safe for long-term use. The anticholinergic antiparkinsonism agent benztropine mesylate aggravated the dyskinesia to a significant degree.

Tardive dyskinesia is now recognised as one of the more severe side effects of antipsychotic medication. Its prevalence has been reported to be as high as $43 \%$ in a sample of psychiatric outpatients (Asnis et al., 1977).

The dyskinesia usually occurs in patients who are still taking antipsychotic medication and who have had several years of treatment, although Crane (1973) found that up to $40 \%$ of patients developed dyskinesia on the cessation of treatment, and Degkwitz et al. (1967) found that in $3.6 \%$ of patients the condition began within six months of starting phenothiazine treatment.

The dyskinesia in most cases is responsive to changes in dosage. Increasing the dosage may relieve the dyskinesia but presumably at the risk of aggravating its underlying pathophysiology. Decreasing the dosage may also alleviate the dyskinesia-Quitkin et al. (1977) found that in 11 out of 12 patients symptoms remitted some weeks or months after the medication was decreased or discontinued but in many cases this was at the expense of an exacerbation of the psychosis. If antipsychotic medication has to be continued it would seem rcasonable to give the smallest dosage necessary to control the psychosis and to use adjunctive therapy to control the dyskinesia.

Address for reprint requests: Dr J. Sydney Smith, Neuropsychiatric Institute, Prince Henry Hospital, PO Box 233, Matraville, NSW 2036, Australia.

Accepted 21 December 1978
Anticholinergic antiparkinsonism agents ares generally held to aggravate tardive dyskinesia and possibly to contribute to its pathogenesis (Kiloho et al., 1973) though cases have been reported in whom the dyskinesia responded favourably to anticholinergic agents (Quitkin et al., 1977).

Thiopropazate hydrochloride (Dartalan) is com monly used in the treatment of tardive dyskinesia. The effectiveness of three weeks of treatment with the drug has been demonstrated in two doubleblind cross-over placebo controlled studies, 23 patients in one study (Singer and Cheng, 1971) and two in the other (Roxburgh, 1970).

This trial was designed to assess the effect of the anticholinergic agent benztropine mesylate (Cogentin) on phenothiazine-induced dyskinesia, to determine the efficacy of long-term treatment of phenothiazine-induced dyskinesia with thiopropazate hydrochloride, and to determine if on withdrawing this drug the severity of dyskinesia was likely to exceed the level found when all medication was withdrawn before the administration of thiopropazate hydrochloride.

\section{Methods}

Patients who had been taking phenothiazines for at least 12 months and who had developed tardive dyskinesia were sought. Patients were excluded from the trial if they were taking any other neuroleptic medication, or had impaired liver function, 
phenothiazine-induced ocular abnormalities, organic disorders known to cause involuntary movements, or a family history suggestive of Huntington's disease.

In the first phase of the trial the patients received benztropine mesylate along with their usual phenothiazine for one month. A cinematographic film was made of their dyskinesia at the end of this period. Then benztropine mesylate was withdrawn, and two weeks later a second film was made. The phenothiazine was stopped for one month before the third film was made. The patients then received thiopropazate hydrochloride $30 \mathrm{mg}$ daily in divided dosage, and a fourth film was made after one month on this medication. A fifth film was made after three months of medication, and a sixth film after six months of medication. Thiopropazate hydrochloride was then withdrawn for a further month and a seventh film made. The assessments were stopped if the patient's dyskinesia resolved or abated substantially during the one month therapy-free period or if for medical or psychiatric reasons it was felt that continuation in the trial was unwarranted. If the patient's psychosis warranted further treatment during the trial, provision was made for the original phenothiazine to be given at a dosage not exceeding that used before the beginning of the trial.

At each stage of the trial the patients were filmed for 90 seconds through a one-way screen. For 60 seconds the face was filmed when the patient was unaware, and for 30 seconds any tongue movements were filmed with the mouth open. The first film for each patient was transferred onto a videotape loop. The other films were assembled in a random order.

To evaluate the severity of the dyskinesia at different stages of the trial, two raters made independent assessments for each patient by comparing the videotape of the first film with other films viewed in a random order. The scoring used was: $0=$ symptom-free, $1=$ great improvement, $2=$ moderate improvement, $3=$ slight improvement, $4=$ no change, $5=$ slightly worse, $6=$ markedly worse. The raters' scores were totalled for each film of the patient before the order in which the film was viewed was revealed. The first film of each patient was scored 4 automatically by each observer, giving a total score of 8 . The correlation between the scores of the two observers was 0.88 .

The mean total scores obtained for the various stages of the trial were compared to determine the effect of withdrawing antiparkinsonism medication, to compare the effect of withdrawing all drugs at the beginning and end of the trial, and to evaluate the efficacy of thiopropazate hydrochloride therapy after one, three, and six months of treatment. The mean total scores were compared using Student's paired $t$ test.

Because of a considerable number of practical problems the drug regime was completed in only a small number of patients. The various alterations to the patients' medication necessitated an inpatient stay of at least six weeks. This precluded the inclusion of outpatients. Long-term inpatients were often too negativistic to be considered for the trial, and a considerable proportion of those who took part became too disturbed on the withdrawal of their usual medication to complete the trial. Because of the small number of patients studied, changes in the severity of their dyskinesia had to be dramatic to reach statistical significance.

\section{Results}

Of the 21 patients admitted to the study, 11 had to be withdrawn before the completion of the trial. The reasons for withdrawal are given in Table 1. Most patients were withdrawn because

Table 1 Outcome in 21 patients who entered the trial

\begin{tabular}{ll}
\hline Outcome & Number \\
& of \\
patients
\end{tabular}

Completed six months of therapy with

thiopropazate hydrochloride

10

Completed three months of therapy with

thiopropazate hydrochloride

Withdrawn from trial because of active psychosis

Withdrawn from trial for medical reasons

5

4

Completed less than three months on thiopropazate hydrochloride

Withdrawn from trial because of active psychosis

Refused to co-operate

Withdrawn before commencement of thiopropazate hydrochloride

Dyskinesia ceased in drug-free interval

Grand mal seizure occurred

of a deterioration of their psychosis but one was withdrawn before starting on thiopropazate hydrochloride when he suffered a grand mal seizure, and another after three months of treatment with thiopropazate hydrochloride when a carcinoma of the colon was diagnosed. The result for the 10 patients who completed six months of treatment with thiopropazate hydrochloride as well as a further five patients who completed three months of treatment have been analysed (Table 2). All but one of these 15 patients were men, and the mean age was 56.5 years with a range of 39 to 69 years. 
Table 2 Total scores for severity of dyskinesia

\begin{tabular}{|c|c|c|c|c|c|c|c|}
\hline \multirow[t]{3}{*}{ Case number } & \multicolumn{7}{|c|}{ Treatment regime } \\
\hline & \multirow{2}{*}{$\begin{array}{l}\text { Phenothiazine } \\
\text { and benztropine } \\
\text { mesylate }\end{array}$} & \multirow{2}{*}{ Phenothiazine } & \multirow[t]{2}{*}{ Drug-firee } & \multicolumn{3}{|c|}{$\begin{array}{l}\text { Thiopropazate hydrochloride } \\
\text { Length of treatment }(\mathrm{mo})\end{array}$} & \multirow[t]{2}{*}{ Drug-free } \\
\hline & & & & One & Three & Six & \\
\hline $\begin{array}{r}1 \\
2 \\
3 \\
4 \\
5 \\
6 \\
7 \\
8 \\
9 \\
10\end{array}$ & $\begin{array}{l}8 \\
8 \\
8 \\
8 \\
8 \\
8 \\
8 \\
8 \\
8 \\
8\end{array}$ & $\begin{array}{r}0 \\
3 \\
6 \\
4 \\
3 \\
8 \\
10 \\
7 \\
5 \\
8\end{array}$ & $\begin{array}{r}0 \\
6 \\
9 \\
9 \\
5 \\
10 \\
6 \\
4 \\
4 \\
5\end{array}$ & $\begin{array}{r}4 \\
2 \\
10 \\
8 \\
10 \\
4 \\
1 \\
3 \\
2 \\
1\end{array}$ & $\begin{array}{l}3 \\
4 \\
3 \\
9 \\
8 \\
8 \\
4 \\
2 \\
4 \\
2\end{array}$ & $\begin{array}{l}2 \\
1 \\
0 \\
3 \\
2 \\
6 \\
6 \\
0 \\
0 \\
3\end{array}$ & $\begin{array}{r}4 \\
4 \\
12 \\
3 \\
6 \\
12 \\
2 \\
5 \\
6 \\
-\end{array}$ \\
\hline $\begin{array}{l}\text { Mean total } \\
\text { score }\end{array}$ & $8.0(n=10)$ & $5.4(n=10)$ & $5.8(n=10)$ & $4.5(n=10)$ & $4.7(n=10)$ & $2.3(n=10)$ & $6.0(n=9)$ \\
\hline $\begin{array}{l}11 \\
12 \\
13 \\
14 \\
15\end{array}$ & $\begin{array}{l}8 \\
8 \\
8 \\
8 \\
8\end{array}$ & $\begin{array}{l}9 \\
7 \\
2 \\
3 \\
9\end{array}$ & $\begin{array}{r}3 \\
8 \\
8 \\
7 \\
12\end{array}$ & $\begin{array}{r}4 \\
7 \\
3 \\
6 \\
10\end{array}$ & $\begin{array}{r}2 \\
9 \\
4 \\
10 \\
9\end{array}$ & & \\
\hline $\begin{array}{l}\text { Mean total } \\
\text { score }\end{array}$ & $8.0(n=15)$ & $5.6(n=15)$ & $6.4(n=15)$ & $5.0(n=15)$ & $5.4(n=15)$ & & \\
\hline
\end{tabular}

For three patients the dosage of thiopropazate hydrochloride had to be reduced during the trial because they developed signs of Parkinsonism. For case 1 the dosage was reduced to $15 \mathrm{mg}$ daily on the tenth day but increased to $30 \mathrm{mg}$ daily after the first month. Case 4 had the dosage reduced to $15 \mathrm{mg}$ daily after two weeks of treatment, and remained on this dosage. Case 6 had the dosage reduced to $20 \mathrm{mg}$ daily at the end of the first month and to $15 \mathrm{mg}$ daily at the end of the second month, and remained on this dosage. Two patients, cases 9 and 11, received other phenothiazine medication during their treatment with thiopropazate hydrochloride.

Of the 15 patients, 10 improved with the cessation of benztropine mesylate while two were judged to be the same, and three worse. The mean total score for the dyskinesia fell from 8.0 when on combined phenothiazine and benztropine mesylate therapy to 5.6 when the benztropine mesylate was withdrawn. This improvement was significant $(t=3.06, \mathrm{df}=14, \mathrm{P}<0.01)$.

Subsequently, when the phenothiazine was withdrawn for a period of one month, nine patients showed an aggravation of the dyskinesia while five showed slight improvement, and one remained the same. The increase in the mean total score was not significant.

The 15 patients were then given thiopropazate hydrochloride and eight of them improved both after one month and three months' treatment, some to a dramatic degree, while only one patient was worse at both of these assessments. The mean total scores obtained at one month and three months did not differ significantly from those obtained during the pretreatment drug-free interval.

Eight of the 10 patients who received six months of therapy with thiopropazate hydrochloride were improved with this treatment, three being symptom-free. One patient appeared to be unchanged while another (case 1) appeared to be worse. The latter patient was observed to have dyskinesia during the period on phenothiazine and during the pretreatment drug-free interval but these movements ceased momentarily during filming. For the 10 patients the mean total score obtained after six months (2.3) was significantly less than that obtained during the pretreatment drug-free interval (5.8) $(t=3.85$, $\mathrm{df}=9, \mathrm{P}<0.01$ ).

Nine patients were filmed one month after the completion of the trial and the cessation of thiopropazate hydrochloride. For these patients the mean total score for the pretreatment drug-free phase (5.9) was almost similar to that obtained during the post-treatment drug-free phase (6.0); the changes in the patients' scores were not statistically significant. For these nine patients the mean score obtained after six months of thiopropazate hydrochloride therapy (2.2) was considerably less than that obtained one month after the cessation of the drug and the deterioration of the patients' scores was significant $(t=2.56, \mathrm{df}=$ $8, \mathrm{P}<0.05)$. 


\section{Discussion}

Tardive dyskinesia is thought to result from an imbalance between dopaminergic and cholinergic influences on the corpus striatum (Klawans, 1973). The dyskinesia after antipsychotic medication is thought to result from a reduction of dopaminergic transmission and a compensatory increase in sensitivity of the receptors. Once established, the dyskinesia can be alleviated by dopamine antagonists or acetylcholine agonists and aggravated by dopamine agonists and acetylcholine antagonists (Gerlach et al., 1974), though Casey (1976) and Carroll et al. (1977) have reported patients in whom the opposite responses were obtained, suggesting that subtypes might exist.

Thiopropazate hydrochloride, being a phenothiazine, presumably suppresses dyskinesia by increasing further the dopaminergic blockade in the striatum. In this long-term evaluation of thiopropazate hydrochloride it was found that the drug produced a small improvement in the mean scores for tardive dyskinesia in the group of 15 patients after one and three months of treatment but a significant improvement in the mean scores for the group of 10 patients after six months of treatment. With the subsequent cessation of the drug, although a deterioration in the mean score occurred, the severity of the dyskinesia did not appear to differ from that observed before treatment began.

Six patients failed to complete the trial because of a severe exacerbation of the psychosis which was not controlled by reintroducing their usual antipsychotic medication, while another patient completed the trial after resuming his previous medication.

Thiopropazate hydrochloride in doses up to $30 \mathrm{mg}$ daily would appear to be an effective agent in controlling the symptoms of tardive dyskinesia, at least over a six month period, without aggravating its underlying pathophysiology. In view of its unsatisfactory antipsychotic activity at this dosage and the considerable risk of Parkinsonism with higher doses (Singer and Cheng, 1971), we suggest that its main value is as an adjunctive agent in patients who need to continue other antipsychotic medication.

Gerlach et al. (1977) reported that the anticholinergic antiparkinsonism drug biperidin aggravated the dyskinesia in all seven patients studied, the changes in the mean dyskinesia score being statistically significant. In our study no patient appeared to benefit to any useful degree from benztropine mesylate therapy, and the mean total score for dyskinesia improved significantly when this medication was withdrawn.

These findings support the hypothesis that tardive dyskinesia may result from dopaminergic hyperactivity and cholinergic hypoactivity in the corpus striatum.

We wish to thank the many doctors who have contributed to this study, and in particular Dr Donald Grant. This study was designed in conjunction with Searle Laboratories. The considerable assistance of their staff and in particular of Mr W. A. Van De Water, is greatly appreciated.

\section{References}

Asnis, G. M., Leopold, M. A., Duvoisin, R. C., and Schwartz, A. H. (1977). A summary of tardive dyskinesia in psychiatric outpatients. American Journal of Psychiatry, 134, 1367-1370.

Carroll, B. J., Curtis, G. C., and Kokmen, E. (1977). Paradoxical responses to dopamine agonists in tardive dyskinesia. American Journal of Psychiatry, 134, 785-789.

Casey, D. E. (1976). Tardive dyskinesia: are there subtypes? New England Journal of Medicine, 295, 1078.

Crane, G. E. (1973). Persistent dyskinesia. British Journal of Psychiatry, 122, 395-405.

Degkwitz, R., Binsack, K. F., Herkert, H., Luxenburger, O., and Wenzel, W. (1967). Zum probleme der persistierend hyperkinesen nach langfristiger anwendung von neuroleptika. Nervenarzt, 38, 170174.

Gerlach, J., Reisby, N., and Randrup, A. (1974). Dopaminergic hypersensitivity and cholinergic hypofunction in the pathophysiology of tardive dyskinesia. Psychopharmacologia (Berlin), 34, 21-35.

Kiloh, L. G., Smith, J. S., and Williams, S. E. (1973). Anti-Parkinson drugs as causal agents in tardive dyskinesia. Medical Journal of Australia, 2, 591593.

Klawans, H. L. (1973). The pharmacology of tardive dyskinesia. American Journal of Psychiatry, 130, 82-86.

Quitkin, F., Rifkin, A., Gochfeld, L., and Klein, D. F. (1977). Tardive dyskinesia: are first signs reversible? American Journal of Psychiatry, 134, 84-87.

Roxburgh, P. A. (1970). Treatment of persistent phenothiazine-induced oral dyskinesia. British Journal of Psychiatry, 116, 277-280.

Singer, K., and Cheng, M. N. (1971). Thiopropazate hydrochloride in persistent dyskinesia. British Medical Journal, 4, 22-25. 